\title{
CODE coefficients based Single frequency Ionospheric Error Correction Model to improve GPS Positional Accuracy of the Low-latitude regions
}

\author{
${ }^{1}$ K. Durga Rao, ${ }^{2}$ Dr. V B S Srilatha Indira Dutt, \\ ${ }^{I}$ Asst. Prof. Dr. L.B.College of Engg. (for women), Visakhapatnam, A.P, INDIA \\ ${ }^{2}$ Assoc. Prof.Dept. of ECE, GITAM University, Visakhapatnam, A.P, INDIA
}

\begin{abstract}
GPS (Global Positioning System) is a satellite based navigation system which provides reliable positioning information to its users (receivers) based on the concept of Time of Arrival (TOA) of the signal at the receiver. The positional accuracy of GPS is affected by several sources such as atmosphere, multipath and satellite-receiver geometry etc., of which ionosphere is one of the major sources of error for single frequency GPS user. When GPS signal propagates through the ionosphere, due to the change in refractive index in atmospheric layers, the signal bends which causes a delay in the arrival of the signal at the receiver. This delay of the signal introduces an error in ranging measurements at the receiver which in turn leads to an error in positioning information. Hence, to improve the positional accuracy, it is necessary estimate the delay of the signal in ionosphere during its transit from satellite to receiver. As this delay is caused by the electron content of the ionosphere, it is also necessary to estimate the Total Electron Content (TEC) in the signal path.

The only way of estimating the ionospheric delay for single frequency receivers is by using Klobuchar algorithm which is based on the GPS broadcast ephemeris data. But this algorithm is optimized for mid latitudes and is very poor in high and low latitude regions. Hence it is required to have a model that is suitable for low latitude regions and near-equatorial regions such as Indian subcontinent. In this paper, low latitude suitable CODE coefficients based Klobuchar algorithm is implemented and is validated.

The paper aims to investigate the performance of the CODE based Klobuchar algorithm by estimating the ionospheric delay using the CODE coefficients for data due to a low-latitude receiver data i.e. IGS station

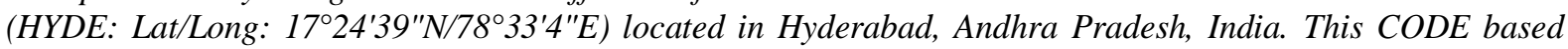
ionospheric delay is compared with delay due to Klobuchar model using the broadcast ephemeris data of the IGS receiver (HYDE). The model performance is validated with the ionospheric delay computed using the IONO-Lab data for the IGS station (HYDE). The CODE based algorithm is implemented, compared and validated for several days data of the IGS receiver (HYDE) and in this paper, it is presented for a typical day i.e. $11^{\text {th }}$ September 2014. The work yields the conclusion that Klobuchar model is simple due to short computation time but better accuracy is obtained using CODE coefficients based Klobuchar model for low latitudinal regions such as Indian subcontinent.
\end{abstract}

Keywords: Total Electron Content, Ionospheric delay, Satellite-Receiver geometry

\section{Introduction}

The Global Positioning System (GPS) is the most well known GNSS and is operated by Department of Defense (U.S.A).The Global Positioning System is a space based navigation system to navigate and relative positioning. GPS was originally developed for radio navigation system but now it is used for many scientific applications.GPS provides opportunity for ionospheric research.GPS consists of a constellation of 24 satellites that tell where the user is in three dimensions[5]. The GPS positional accuracy is affected by several sources; one such source is atmosphere which causes refraction of GPS signal and produces an error of 1-50m [1].The ionospheric delay is the dominant of all error sources. The ionospheric delay is proportional to total electron content (TEC) along the ray path.TEC describes ionosphere with high temporal resolution.

The ionosphere is the region of atmosphere which is approximately of 50 to $1000 \mathrm{~km}$ above the earth's surface and is characterized by presence of free electrons. As GPS signals passes through ionosphere it faces two delays one is bending of signal and second is slowing of signal this creates problem to GPS single frequency users. The refractive index of ionosphere varies as function of height depending on electron density. The ionosphere is formed mainly by the radiation of the Sun striking the atmosphere of the Earth Ionizing molecules.

In this paper ionospheric delay is estimated for a typical day $11^{\text {th }}$ September 2014 .The delay is estimated by using data provided by(SOPAC) for one of the (IGS) station i.e. Long: $17^{\circ} 24^{\prime} 39^{\prime \prime} \mathrm{N} / 78^{\circ} 33^{\prime} 4^{\prime \prime} \mathrm{E}$ ) located in Hyderabad, Andhra Pradesh, India .Ionospheric delay is computed by Klobuchar model, modified 
Klobuchar model using set of coefficients from CODE (centre for orbit determination) in Europe and IONOLAB TEC.

\section{Code range measurements}

GPS errors can arise from inaccuracies is estimating satellite position, satellite clock corrections, tropospheric ,ionspheric effects along the signal propagation and multipath. These effects are included in the equation below; The ranging equation of the arrived GPS signal can be given as

$$
p=\rho+c(d t-d T)+d_{\text {ion }}+d_{\text {tro }}+\varepsilon_{[1]}
$$

Where ' $\mathbf{p}$ ' is measured pseudo range ; ' $\rho$ ' is geometric (or true) range

'c' represents speed of light; dt and dT are offsets of satellite and receiver clocks

' $d_{\text {ion }}{ }^{\prime}, ' d_{\text {tro }}$ ' are delays due to ionosphere and troposphere

' $\varepsilon$ ' is effects of multipath and receiver measurement noise

\section{Estimation of Ionospheric delay}

The ionospheric delay is a function of Total Electron Content (TEC)which is frequency dependent.TEC is one square meter column along the signal propagation path starting from satellite to the receiver. The nature of ionosphere is dispersive which allows calculation of the absolute TEC, if range measurements on 2 different frequencies L1 (1575.42 MHZ) and L2 (1227.6 MHZ) and if the arrival times of these 2 frequencies, which are different at the Receiver, is known then ionospheric delay is found.TEC can estimated by differencing the 2 code range measurements on L1 and L2 or 2 phase measurements.

\subsection{Total Electron Content}

The TEC is important in determining delay of GPS signal in ionosphere and is defined as the total number of electrons present in an area of $1 \mathrm{~m}^{2}$ cross section along the line sight of the satellite to receiver. TEC is defined as the integral of the electron density along the propagation path of the signal or the numbers of free electrons in a column of unit cross sectional.

$\mathrm{TEC}=\int_{\mathrm{p}} \mathrm{N}(\mathrm{s}) \mathrm{ds}$

where, $\mathrm{N}(\mathrm{s})$ is the electron content per unit volume and $\mathrm{p}$ is the propagation path between the source and the receiver. TEC is measured in TECU, which indicates $10^{16}$ electrons in an area of $1 \mathrm{~m}^{2}$.

\subsection{Klubochar Algorithm}

The klobuchar model is for ionospheric delay estimation of the single frequency GPS receivers. In this model, ionospheric delay is estimated by considering the TEC between the satellite and the receiver, and 50 to $60 \%$ of the error can be eliminated based on the solar activity of the region. In this, it is assumed that the electron density is more at an altitude of $350 \mathrm{Km}$ on an imaginary line of thickness zero and is maximum at 14:00Hrs of the local time. The klubochar model makes use of geomagnetic latitude on Ionospheric Pierce Point(IPP), which is defined as the point where the line of sight connecting the satellite and receiver meets the single layer at an altitude of $350 \mathrm{Km}[6]$.In order to estimate delay due to klubochar model, klubochar coefficients $\alpha_{n}$ and $\beta_{n}$ are required. The GPS master control station broadcast these coefficients in GPS navigation message. The $\alpha_{n}$ and $\beta_{n}$ are collected from the navigation data of the IGS station NGRI, Hyderabad, on the typical day i.e. September $11^{\text {tht }} 2014$.

Steps to calculate the delay using klubochar algorithm are:

a. Calculate the Earth-centered angle using the Elevation angle of the satellites w.r.t ground station using Eq.1

$$
\begin{aligned}
& \psi=\frac{0.0137}{(E+0.11)}-0.22 \\
& \phi_{i}=\phi_{u}+\psi \cos A \\
& \lambda_{I}=\lambda_{U}+\left(\psi \frac{\sin A}{\cos \left(\phi_{I} \times 3.14\right)}\right) \\
& \phi_{\mathrm{m}}=\phi_{I}+0.064 \cos \left[\left(\lambda_{I}-1.617\right) \times 3.14\right] \\
& t=4.32 * 10^{4} \lambda_{I}+\text { Time }_{G P S} \\
& S F=1+16(0.53-E)^{3}
\end{aligned}
$$

$$
x=\frac{2 \pi(t-50400)}{P E R}
$$


$A M P=\sum_{i=0}^{3} \alpha_{n} \phi_{n}^{n}$

if $|x|>1.57$ then use $T_{\text {iono }}=S F \times\left(5 \times 10^{-9}\right)$

Otherwise $T_{\text {iono }}=S F \times\left[\left(5 \times 10^{-9}\right)+A M P \times\left(1-\frac{x^{2}}{2}+\frac{x^{4}}{4}\right)\right]$ From this ionospheric time delay range delay on any one of the two carriers can be calculated.

\subsection{SOPAC}

\section{Sources Of Data}

Scripps Orbit And Permanent Array Center (SOPAC) for one of the International GNSS Service (IGS)

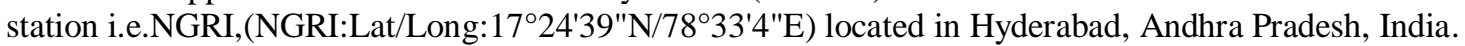

\subsection{IONOLAB}

IONOLAB is used to estimate TEC values for a whole day or a desired time period both for quiet and disturbed days of the ionosphere. It provides TEC estimates for high-latitude, mid-latitude and equatorial stations. Signals from the low elevation satellites are prone to multipath effects. In order to reduce the distortion due to multipath signals, the optimum weighting function is implemented in IONOLAB, minimizing the nonionospheric noise effects. GPS receivers record both pseudorange and phase data of signals. IONOLAB can input TEC computed from the pseudorange measurements or phase-corrected noise. The TEC estimates for both of these inputs are in good. Therefore by taking either pseoudorange or phase-corrected data input, high resolution, accurate TEC estimates is be obtained from IONOLAB.

\subsection{CODE}

CODE stands for centre for orbit determination in Europe.

\section{Results}

The Results are based on the data due to the dual frequency GPS Receiver located at National Geophysical Research Institute, Hyderabad (Hyde: Long/Lat: 78 $33^{\prime} 4^{\prime \prime E} 17^{\circ} 24^{\prime} 39^{\prime \prime N}$ ). The data of a typical day i.e September 11th 2014 is collected and sampled at an interval of 30 seconds, in order to carry out the work.

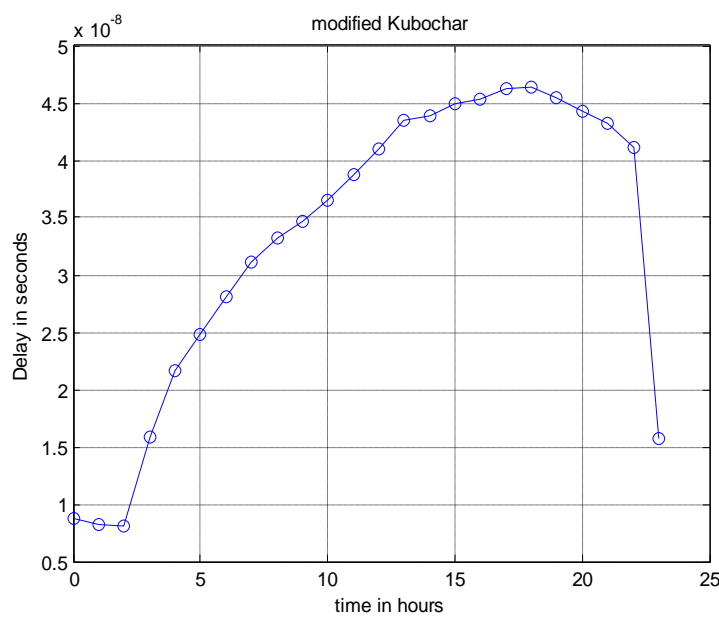



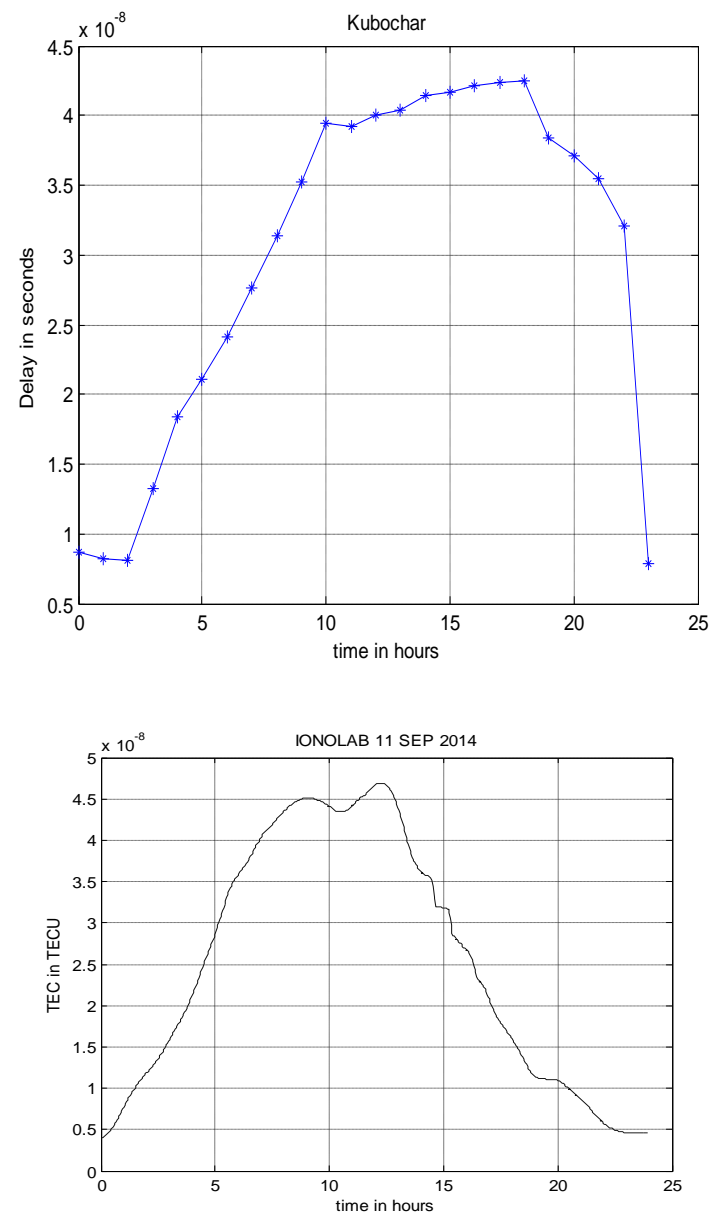

\section{Conclusion}

Ionosphere Delay is estimated using Klobuchar Algorithm (Single Frequency Receiver) and ,modified klobuchar algorithm using CODE coefficients and using IONOLAB TEC for a complete day i.e $11^{\text {th }}$ September 2014 for (NGRI) National Geophysical Research Institute,hyde site and observed that the effect of Ionosphere delay on GPS signal is more in mid afternoon of the day as compared to pre sunrise and post sunset of the day .

The work yields the conclusion that Klobuchar model is simple due to short computation time but better accuracy is obtained using CODE coefficients based Klobuchar model for low latitudinal regions such as Indian subcontinent. The model performance is validated with the IONO-LAB data for the HYDE station .

\section{References}

[1]. Kaplan, E. D., ed., 'Understanding GPS Principles and Applications', Artech House, Norwood, MA, 1996

[2]. Spilker JJ, Parkinson BW (Eds) 'Global positioning system: theory and applications', vol 1. AIAA, pages $485-515$.

[3]. Chen, K. and Gao, Y. (2005). An Ionospheric Effect Estimation Model for Single-Frequency Precise Point Positioning”'

[4]. Liu, J.Y., Chuo, Y.J., Shan, S.J., Tsai, Y.B., Pulinets, S.A. and Yu, S.B. (2004) Pre-Earthquake Ionospheric Anomalies Monitored by GPS TEC. Annals of Geophysics

[5]. G.S.Rao, 'Global Navigation Satellite Systems', Tata McGraw Hill publications, 2010

[6]. Klobuchar, [J.A. 1987] 'Ionospheric time-delay algorithm for single-frequency GPS users', IEEE Trans Aerospace Electron Syst., 23 (3), pages 325-331.

[7]. Pulinets, S.A., Leyva, C.A., Bisiacchi-Giraldi, G. and Ciraolo, L. (2005) Total Electron Content Variations in the Ionosphere before the Colima, Mexico, Earthquake of 21 January 2003. Geofisica International, 44, 369-377.

[8]. Wielgosz, P.; Baran, L.W.; Shagimuratov, I.I.; Aleshnikova, M.V. 2004. Latitudinal Variations of TEC over Europe Obtained from GPS Observations, Annales Geophysicae 22: 405-415. http://dx.doi.org/10.5194/angeo-22-405-2004

[9]. Klobuchar, J.A., "Ionospheric corrections for the single frequency user of the global positioning system". National telesystems Conference, NTC 82 Systems for the Eighties, Galveston, Texas, USA (New York, IEEE, 1982). 